\title{
Draft Genome Sequence of Diaporthe batatatis Causing Dry Rot Disease in Sweetpotato
}

\author{
Yiling Yang, Xiaojian Yao, Xinxin Xhang, Hongda Zou, JingYi Chen, Boping Fang, and \\ Lifei Huang ${ }^{\dagger}$
}

Guangdong Provincial Key Laboratory of Crop Genetic Improvement, Crops Research Institute, Guangdong Academy of Agricultural Sciences, Guangzhou 510640, China

\begin{abstract}
Dry rot caused by Diaporthe batatatis leads to the serious decay of sweetpotato storage roots during postharvest storage, which can result in considerable economic loss. Genomic research of the pathogen could provide a basis for study and prevention of sweetpotato dry rot. Herein, we report a high-quality draft genome sequence of $D$. batatatis CRI 302-4 isolated from infected sweetpotato storage roots in Taizhou City, Zhejiang Province, China. The size of the genome was $54.38 \mathrm{Mb}$ and consisted of 36 scaffolds with a $\mathrm{G}+\mathrm{C}$ content of $50.56 \%$ and an $\mathrm{N}_{50}$ of $2,950,914 \mathrm{bp}$. The information provided in this genome sequence will be an invaluable resource for molecular genetic research and disease control in sweetpotato production.
\end{abstract}

Dry rot is one of the most common diseases affecting sweetpotato during storage. It is caused by Diaporthe batatatis (Harter \& E. C. Field), which belongs to the subclass Diaporthomycetidae (Sordariomycetes) Diaporthales (Guo et al. 2020; Senanayake et al. 2017; Wang et al. 2021). The disease commonly occurs in Zhejiang, Sichuan, and Guangdong Provinces, China (Huang et al. 2020). The infected storage roots undergo shrinking and wrinkling, with light to dark-brown lesions on the outside and dark-brown to black lesions on the inside (Clark et al. 2013). Decay is usually limited to the end of the storage root, causing it to become firm and dry (Clark et al. 2013). The broad occurrence of the disease can lead to decay of all storage roots in the storage room, resulting in considerable economic losses (Huang et al. 2020). Because the effects are similar and the causal fungi are closely related, dry rot can be difficult to distinguish from foot rot (Clark et al. 2013), which has caused controversy in both the sweetpotato production industry and research. Genome sequencing of the pathogen could be an effective way to ameliorate this problem and provide a basis for the research of sweetpotato dry rot. In 2015, CRI 302-4 was isolated from infected sweetpotato roots in Taizhou City, Zhejiang Province, China. The isolate was identified as $D$. batatatis based on morphological traits and the phylogenetic tree (Fig. 1), which was constructed by the internal transcribed spacer, translation elongation factor $1-\alpha$, histone $\mathrm{H} 3$, and $\beta$-tubulin genes from 31 isolates of Diaporthe spp. (Supplementary Table S1) (Guo et al. 2020) and CRI 302-4, using MEGA7 (Kumar et al. 2016) and SequenceMatrix 1.7.8 (Vaidya et al. 2011) software. In the study presented herein, using CRI 302-4 as representative of the pathogen, we carried out genome sequencing, which resulted in the generation of a high-quality genome sequence for sweetpotato dry rot research.

The isolated $\mathrm{CRI} 302-4$ was cultured with shaking at $120 \mathrm{rpm}$ for 7 days at $28^{\circ} \mathrm{C}$ in liquid nutrient agar ( $\mathrm{pH}$ 6.0). Genomic DNA was extracted using a Qiagen genomic tip-100/G DNA isolation kit (Qiagen Inc., Hilden, Germany) according to the manufacturer's protocol. DNA quality and concentration were assessed using $0.5 \%$ agarose gel electrophoresis and a Qubit

${ }^{\dagger}$ Corresponding author: L. Huang; huanglifei@gdaas.cn

*The $e$-Xtra logo stands for "electronic extra" and indicates that a supplementary table is published online.

The author(s) declare no conflict of interest.
Funding

This research was supported by the Project of Science and Technology of Guangdong Province (2018A050506053), China Agriculture Research System of MOF and MARA, and Sweetpotato Innovation Team of Modern Agricultural Industry Technology System in Guangdong Province (2020KJ111).

\section{Keywords}

Diaporthe batatatis, disease management, dry rot, fungi, genome, sweetpotato

Accepted for publication 6 October 2021

C 2022 The American Phytopathological Society 


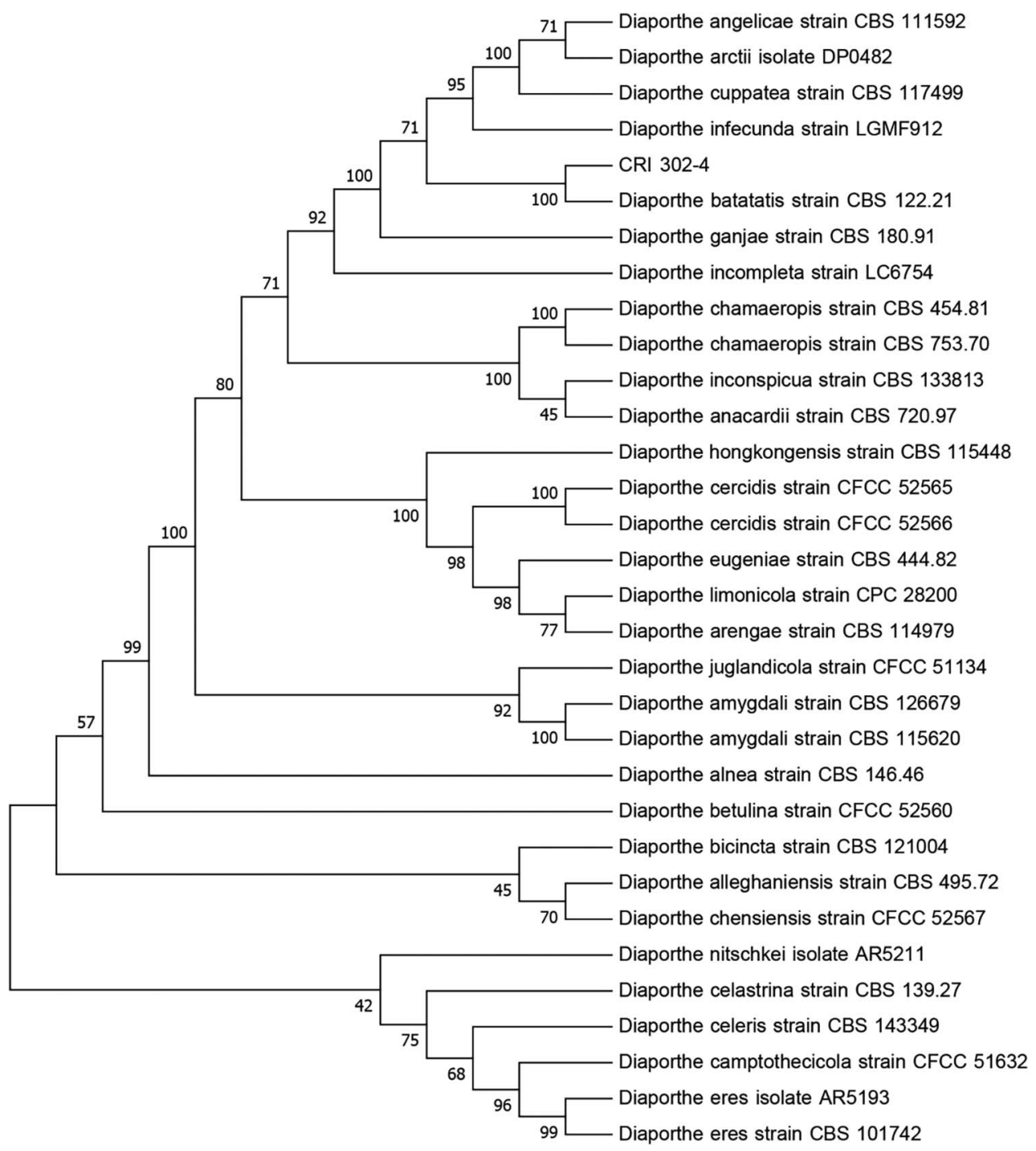

Fig. 1. Phylogenetic analysis of Diaporthe batatatis CRI 302-4 and another 31 isolates of Diaporthe spp.

3.0 fluorimeter (Invitrogen, Carlsbad, CA, U.S.A.). The DNA concentration was $230.0 \mathrm{ng} / \mu \mathrm{l}$, with an absorbance at $260 / 280 \mathrm{~nm}$ ratio of 1.89 . The whole-genome sequencing data were obtained using the Oxford Nanopore Technology PromethION platform (Oxford Nanopore, Oxford, U.K.) and short-read Illumina on an Illumina HiSeq 4000 sequencer.

In total, 1,040,623 raw reads (7.93 Gb) were generated by Nanopore sequencing. After adapter sequences, low-quality bases, and short reads (<2 kb) were filtered out, 739,556 clean reads $\left(7.32 \mathrm{~Gb}\right.$ ) were obtained, with an $\mathrm{N}_{50}$ of $14,757 \mathrm{bp}$, which corresponded to an approximately $134.72 \times$ sequence depth. The clean reads were corrected by Canu v1.5 (Koren et al. 2017) and assembled using wtdbg2 v2.3 (Ruan and Li 2020) with parameters -H -k 21 -j 1000 -S 1.02 -e 3; and Pilon v1.23 (Walker et al. 2014) with default parameters, which was run for five rounds. The genome size was $54.38 \mathrm{Mb}$ and consisted of 36 scaffolds with a $\mathrm{G}+\mathrm{C}$ content of $50.56 \%$ and $\mathrm{N}_{50}$ of $2,950,914 \mathrm{bp}$; the longest scaffold was $7,611,566 \mathrm{bp}$ (Table 1).

The completeness of the genome assembly was assessed by benchmarking universal single-copy ortholog (BUSCO) (Simão et al. 2015) and comparison with short Illumina reads. A paired-end library with an average insert size of $350 \mathrm{bp}$ was constructed following the standard Illumina library construction and sequencing protocol (Illumina, San Diego, CA, U.S.A.). Illumina reads with $91.23 \times$ depth were mapped to the assembled genome using BurrowsWheeler alignment software (Li and Durbin 2009). Approximately $98.44 \%$ of the clean reads mapped to the genome, with genome coverage of $94.81 \%$. Assessment by BUSCO was based on the 290 conserved core genes in the fungi_odb database (Simão et al. 2015), using BUSCO v2.0 software. In all, 284 complete BUSCO genes were found in the assembled 
Table 1. Genome assembly statistics of the assembled Diaporthe batatatis CRI 302-4

\begin{tabular}{lc} 
Feature $^{\text {a }}$ & D. batatatis \\
Depth of Nanopore sequence & $134.72 \times$ \\
Depth of Illumina sequence & $91.23 \times$ \\
Genome size $(\mathrm{Mb})$ & 54.38 \\
Number of scaffolds & 36 \\
$\mathrm{~N}_{50}$ length (bp) & $2,950,914$ \\
$\mathrm{~N}_{90}$ length (bp) & 653,982 \\
Scaffold maximum (bp) & $7,611,566$ \\
G+C content (\%) & 50.56 \\
BUSCO completeness (\%) & 97.93 \\
Repeat rate (\%) & 2.59 \\
Number of predicted protein-coding genes & 14,138 \\
Number of annotated genes & 13,037 \\
Number of nr annotated genes & 12,982 \\
Number of pathogen-host interaction proteins & 4,329 \\
Number of carbohydrate-active enzymes & 941 \\
Number of transport proteins & 141 \\
Number of signal peptides & 1,650 \\
Number of transmembrane proteins & 3,027 \\
Number of secreted proteins & 1,224 \\
Number of effector proteins & 144 \\
GenBank accession number & JAHWGW000000000 \\
\hline
\end{tabular}

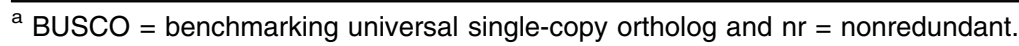

genome, resulting in completeness of $97.93 \%$ (284 of 290), indicating that the genome was appropriately assembled.

To analyze the constitution of the genome, prediction of repeated sequences and proteincoding genes was performed. The repeated sequence database was used to predict the repeated sequences in the pathogenic genome using Repeatmasker v4.0.6 software (Chen 2004), which was constructed using LTR_FINDER v1.05 (Xu and Wang 2007), MIX-Hunter (Han and Wessler 2010), RepeatScout v1.0.5 (Price et al. 2005), and PILER-DF v2.4 (Edgar and Myers 2005) software, according to structure and de novo prediction. In total, 1,410,223bp repeated sequences were detected, accounting for $2.59 \%$ of the assembled genome. Protein-coding genes were predicted using ab initio-, homology-, and RNA sequencing-based methods, which were integrated by EVM v1.1.1 (Haas et al. 2008) and modified by PASA v2.0.2 (Campbell et al. 2006). In total, 14,138 protein-coding genes were obtained, including 41,272 exons and 27,134 Introns.

The predicted genes were aligned to eukaryotic orthologous groups (KOG) (Tatusov et al. 2000), Kyoto Encyclopedia of Genes and Genomes (KEGG) (Kanehisa et al. 2004), SwissProt (Boeckmann et al. 2003), TrEMBL (Boeckmann et al. 2003), nonredundant (nr) (Deng et al. 2006), gene ontology (GO) (Ashburner et al. 2000), and Pfam (Finn et al. 2016) databases. In total, 13,037 genes were functionally annotated, accounting for $92.21 \%$. Among them, 12,982 genes (91.82\%) were functionally annotated in the $\mathrm{nr}$ database. The pathogenicity genes were predicted using the pathogen-host interactions $(\mathrm{PHI})$ database (Winnenburg et al. 2006), which identified 4,329 (30.62\%) putative $\mathrm{PHI}$ genes. Carbohydrate-active enzymes (CAZymes), which play an important role in infection by the breaking down of host cell wall components, were predicted using the CAZy database (Cantarel et al. 2009) and HMMER (Eddy 1998) software. In total, 941 CAZymes were identified, which were further classified into 398 glycoside hydrolases, 121 glycosyl transferases, 33 polysaccharide lyases, 165 carbohydrate esterases, 213 auxiliary activities, and 97 carbohydrate-binding modules. In addition, 141 transport proteins were predicted utilizing the Transporter Classification Database (TCDB) (Saier et al. 2006), 1,650 signal peptides were predicted by SignalP 4.0 (Petersen et al. 2011), 3,027 transmembrane proteins were predicted using TMHMM software (Petersen et al. 2011), 1,224 secreted proteins were predicted by removing the number of predicted transmembrane proteins from the number of predicted signal peptides, and 144 effector proteins were predicted using EffectorP (Petersen et al. 2011).

The study presented herein reports a high-quality draft genome assembly of the pathogen $D$. batatatis, which will serve as a reference genome for molecular genetic marker development, gene cloning, investigation of propagation and evolution mechanisms, and comparative genomics. The described Whole-Genome Shotgun project has been deposited at DNA Data Bank of Japan/ 
European Nucleotide Archive/GenBank under the accession code JAHWGW000000000. The version described in this article is JAHWGW010000000. The $D$. batatatis isolate CRI 302-4 used in this study is available at the Agricultural Culture Collection of China (ACCC) in Beijing and at the Crops Research Institute Guangdong Academy of Agricultural Sciences in Guangzhou, China.

\section{Literature Cited}

Ashburner, M., Ball, C. A., Blake, J. A., Botstein, D., Butler, H., Cherry, J. M., Davis, A. P., Dolinski, K., Dwight, S. S., Eppig, J. T., Harris, M. A., Hill, D. P., Issel-Tarver, L., Kasarskis, A., Lewis, S., Matese, J. C., Richardson, J. E., Ringwald, M., Rubin, G. M., and Sherlock, G. 2000. Gene Ontology: Tool for the unification of biology. Nat. Genet. 25:25-29.

Boeckmann, B., Bairoch, A., Apweiler, R., Blatter, M. C., Estreicher, A., Gasteiger, E., Martin, M. J., Michoud, K., O'Donovan, C., Phan, I., Pilbout, S., and Schneider, M. 2003. The SWISS-PROT protein knowledgebase and its supplement TrEMBL in 2003. Nucleic Acids Res. 31:365-370.

Campbell, M. A., Haas, B. J., Hamilton, J. P., Mount, S. M., and Buell, C. R. 2006. Comprehensive analysis of alternative splicing in rice and comparative analyses with Arabidopsis. BMC Genomics 7:327.

Cantarel, B. I., Coutinho, P. M., Rancurel, C., Bernard, T., Lombard, V., and Henrissat, B. 2009. The Carbohydrate-Active EnZymes database (CAZy): An expert resource for glycogenomics. Nucleic Acids Res. 37:D233-D238.

Chen, N. 2004. Using RepeatMasker to identify repetitive elements in genomic sequences. Curr. Protoc. Bioinf. 4:Unit 4.10.

Clark, C. A., Ferrin, D. M., Smith, T. P., and Holmes, G. J. 2013. Pages 58-59 in: Compendium of Sweetpotato Diseases, Pests, and Disorders, 2nd Ed. The American Phytopathological Society, St. Paul, MN, U.S.A.

Deng, Y. Y., Li, J. Q., Wu, S. F., Zhu, Y., Chen, Y. W., and He, F. C. 2006. Integrated $\mathrm{nr}$ database in protein annotation system and its localization. Comput. Eng. 32:71-72.

Eddy, S. R. 1998. Profile hidden Markov models. Bioinformatics 14:755-763.

Edgar, R. C., and Myers, E. W. 2005. PILER: Identification and classification of genomic repeats. Bioinformatics 21:i152-i158.

Finn, R. D., Coggill, P., Eberhardt, R. Y., Eddy, S. R., Mistry, J., Mitchell, A. L., Potter, S. C., Punta, M., Qureshi, M., Sangrador-Vegas, A., Salazar, G. A., Tate, J., and Bateman, A. 2016. The Pfam protein families database: Towards a more sustainable future. Nucleic Acids Res. 44:D279-D285.

Guo, Y. S., Crous, P. W., Bai, Q., Fu, M., Yang, M. M., Wang, X. H., Du, Y. M., Hong, N., Xu, W. X., and wang, G. P. 2020. High diversity of Diaporthe species associated with pear shoot canker in China. Persoonia Mol. Phylogeny Evol. Fungi. 45:132-162.

Haas, B. J., Salzberg, S. L., Zhu, W., Pertea, M., Allen, J. E., Orvis, J., White, O., Buell, C. R., and Wortman, J. R. 2008. Automated eukaryotic gene structure annotation using EVidenceModeler and the Program to Assemble Spliced Alignments. Genome Biol. 9:R7.

Han, Y., and Wessler, S. R. 2010. MITE-Hunter: A program for discovering miniature inverted-repeat transposable elements from genomic sequences. Nucleic Acids Res. 38:e199.

Huang, L., Fang, B., Chen, J., Zhang, X., Luo, Z., Yang, Y., et al. 2020. Pages 68-70 in: Sweetpotato Diseases Identification and Control Guide. China Agriculture Press, Beijing, China.
Kanehisa, M., Goto, S., Kawashima, S., Okuno, Y., and Hattori, M. 2004. The KEGG resource for deciphering the genome. Nucleic Acids Res. 32:D277-D280.

Koren, S., Walenz, B. P., Berlin, K., Miller, J. R., Bergman, N. H., and Phillippy, A. M. 2017. Canu: Scalable and accurate long-read assembly via adaptive k-mer weighting and repeat separation Sergey. Genome Res. 27:722-736.

Kumar, S., Stecher, G., and Tamura, K. 2016. MEGA7: Molecular Evolutionary Genetics Analysis version 7.0 for bigger datasets. Mol. Biol. Evol. 33:1870-1874.

$\mathrm{Li}$, H., and Durbin, R. 2009. Fast and accurate short read alignment with Burrows-Wheeler transform. Bioinformatics 25:1754-1760.

Petersen, T. N., Brunak, S., Von Heijne, G., and Nielsen, H. 2011. SignalP 4.0: Discriminating signal peptides from transmembrane regions. Nat. Methods 8: 785-786.

Price, A. L., Jones, N. C., and Pevzner, P. A. 2005. De novo identification of repeat families in large genomes. Bioinformatics 21:i351-i358.

Ruan, J., and Li, H. 2020. Fast and accurate long-read assembly with wtdbg2. Nat. Methods 17:155-158.

Saier, M. H., Tran, C. V., and Barabote, R. D. 2006. TCDB: The Transporter Classification Database for membrane transport protein analyses and information. Nucleic Acids Res. 34:D181-D186.

Senanayake, I. C., Crous, P. W., Groenewald, J. Z., Maharachchikumbura, S. S. N., Jeewon, R., Phillips, A. J. L., Bhat, J. D., Perera, R. H., Li, Q. R., Li, W. J., Tangthirasunun, N., Norphanphoun, C., Karunarathna, S. C., Camporesi, E., Manawasighe, I. S., Al-Sadi, A. M., and Hyde, K. D. 2017. Families of Diaporthales based on morphological and phylogenetic evidence. Stud. Mycol. 86:217-296.

Simão, F. A., Waterhouse, R. M., Ioannidis, P., Kriventseva, E. V., and Zdobnov, E. M. 2015. BUSCO: Assessing genome assembly and annotation completeness with single-copy orthologs. Bioinformatics 31:3210-3212.

Tatusov, R. L., Galperin, M. Y., Natale, D. A., and Koonin, E. V. 2000. The COG database: A tool for genome-scale analysis of protein functions and evolution. Nucleic Acids Res. 28:33-36.

Vaidya, G., Lohman, D. J., and Meier, R. 2011. SequenceMatrix: Concatenation software for the fast assembly of multi-gene datasets with character set and codon information. Cladistics 27:171-180.

Walker, B. J., Abeel, T., Shea, T., Priest, M., Abouelliel, A., Sakthikumar, S., Cuomo, C. A., Zeng, Q., Wortman, J., Young, S. K., and Earl, A. M. 2014. Pilon: An integrated tool for comprehensive microbial variant detection and genome assembly improvement. PLoS One 9:e112963.

Wang, X., Dong, H., Lan, J., Liu, Y., Liang, K., Lu, Q., Fang, Z., and Liu, P. 2021. High-quality genome resource of the pathogen of Diaporthe (Phomopsis) phragmitis causing kiwifruit soft rot. Mol. Plant-Microbe Interact. 34:218-221.

Winnenburg, R., Baldwin, T. K., Urban, M., Rawlings, C., Köhler, J., and HammondKosack, K. E. 2006. PHI-base: A new database for pathogen host interactions. Nucleic Acids Res. 34:D459-D464.

$\mathrm{Xu}, \mathrm{Z}$., and Wang, H. 2007. LTR-FINDER: An efficient tool for the prediction of full-length LTR retrotransposons. Nucleic Acids Res. 35:W265-W268. 